\title{
Plastic pollution and World Environment Day 2018
}

\section{Editorial}

We are celebrating the World Environment Day (WED) on $5^{\text {th }}$ June 2018 to encourage the alertness for the protection of our envirome. The world environment day is the great day to celebrate about the awareness among the common people about our environment and its significance in term of sustainability, effects of changing climate, impact of various pollution and various activities done by the scholars in the world towards the environmental protection. The world environment day is widely celebrated across the world and it was first established by United Nations Environment Program (UNEP) in 1972. Each WED is organized about a theme that emphases the consideration on a predominantly persistent to environmental apprehension. This year, India is the host country. The theme for this year 2018 is beating plastic pollution. Plastic is synthetic substance made of organic polymers that can be molded into different shapes. Plastic is used everywhere in different form start from our house to ships, aero planes, even the healthcare industry would not be possible without use of plastic material. Natural plastic is also found from rubber tree. Jambeck ${ }^{1}$ reported that $\sim 8,000,000$ tons per annum of plastic goes into the ocean. ${ }^{1}$ Eriksen et al., ${ }^{2}$ estimated that 525,000,000 pieces of plastic are present in the sea. ${ }^{2}$ It is estimated in 2010 that approximately $1 \%$ of global plastic waste enter in to sea. ${ }^{3}$ This plastic adsorbs deadly chemicals like PCBs and DDTs, increasing their concentration (accumulation) by a million. ${ }^{4}$ These stubborn pollutants enter into biomass and hence bioaccumulation takes place in the food chain which results higher concentration of pollutants in fish ${ }^{5}$ including other species which ultimately reach to humans.

Avio et al., ${ }^{6}$ identified large-scale junction points of plastic debris. Surveys on time-based drifts, geographical dissemination and global succession of plastics have management implications when defining the origin, possible drifting tracks and ecological consequences of such pollution. The number of marine species is known to be affected by plastic adulteration. Close to imbroglio and assimilation of macro-debris by large vertebrates, micro-plastics are accumulated by planktonic and invertebrate organisms, being transferred along food chains. The adverse consequences include loss of nutritional value of diet, physical damages, exposure to pathogens and transport of alien species. China is the country where most $(27.7 \%$ of global mismanaged plastic waste) of the plastic waste is mismanaged i.e. 8.82 million tons per annum. ${ }^{1}$

The sea plastic can degrade into smaller pieces by the effects of sunlight, oxidation and the abrasion of waves and currents, becoming smaller and smaller often to the point they are no longer visible to the naked eye. Marine organisms consume the micro-plastics as food by mistake. The consumption of plastic by marine-organism increases persistent, bio-accumulative and toxic substances to the aquatic food chain. However, it is not clear what the net effect of plastic wastes may be in either transferring stubborn pollutants or reducing their bioavailability. As per Leonard of Ocean Conservancy, the oceanic plastic pollution will remain a difficult problem to solve because it represents a fundamental market failure on a worldwide scale. The benefits of citizen-focused activities such as beach cleaning are well recognized from an educational as well as a litter-removal perspective. ${ }^{7}$ However, there are concerns about the effectiveness and practicality of large-scale mechanical clean-up operations at sea. This is because; current rates of entry for jumble to the marine environment
Volume 3 Issue 3 - 2018

\author{
Ashok K Rathoure \\ Ecology \& Biodiversity Expert, Ecosystem Resource \\ Management Pvt. Ltd., India
}

Correspondence: Ashok K Rathoure, Ecology \& Biodiversity Expert, Ecosystem Resource Management Pvt. Ltd., Hardoi-24I00I (UP), India, India, Tel +9194 5050 I47I, Email asokumr@gmail.com

Received: June 04, 2018 | Published: June 05, 2018

far surpass the probable for removal by clean-up approach. Hopewell et al., ${ }^{8}$ proposed the plastic for re-uses or re-manufacturing of plastic components in goods. If the plastic can be reused, reduced at source, and re-manufactured in various goods, hence we can decrease the load of plastic pollution in the environment. The plastic pollution should be stopped and re-think on use of plastic and its items. Individual can also contribute in the reduction of plastic waste generation by avoiding the plastic-bottled beverages, minimal use of plastic products, use of re-usable shopping bags, use of natural fiber bags, etc. There is an urgent need of awareness on the fate and impact of plastic waste among the public.

\section{Acknowledgements}

None.

\section{Conflict of interest}

Author declares that he has no conflict of interest.

\section{References}

1. Jambeck Jenna R. Plastic waste inputs from land into the ocean. Science. 2015;(347):768.

2. Eriksen M, Lebreton LCM, Carson HS, et al. Plastic Pollution in the World's Oceans: More than 5 Trillion Plastic Pieces Weighing over 250,000 Tons Afloat at Sea. PLoS ONE. 2014;9(12):e111913.

3. Cozar A, Echevarria F, Gonzales Gordillo I, et al. Plastic debris in the open ocean. USA: Proc Natl Acad Sci; 2014.

4. Yukie Mato, Tomohiko Isobe, Hideshige Takada, et al. Plastic Resin Pellets as a Transport Medium for Toxic Chemicals in the Marine Environment. Environmental Science and Technology. 2001;35(2):318324.

5. TanakaKosuke, Hideshige Takada, Rei Yamashita, et al. Accumulation of plastic-derived chemicals in tissues of seabirds ingesting marine plastics. Mar Pollut Bull. 2013;69(1-2):219-222.

6. Avio CG, Stefania Gorbi, Francesco Regoli. Plastics and micro plastics in the oceans: From emerging pollutants to emerged threat. Mar Environ Res. 2017;128:2-11.

7. Nelms SE, Coombes C, Foster LC, et al. Marine Anthropogenic Litter on British beaches: A 10-year Nationwide Assessment using Citizen Science Data. Science of the Total Environment. 2017;(579):1399-1409.

8. Hopewell J, Dvorak R, Kosior E. Philosophical Transactions of the Royal Society B: Biological Sciences. Plastics recycling: challenges and opportunities. 2009;(364):2115-2126. 